\title{
CD10 immunohistochemistry stains enteric mucosa, but negative staining is unreliable in the setting of active enteritis
}

\author{
Joshua M Lloyd ${ }^{1}$ and Scott R Owens ${ }^{2}$ \\ ${ }^{1}$ Department of Pathology, The University of Pittsburgh Medical Center, Pittsburgh, PA, USA \\ and ${ }^{2}$ Department of Pathology, The University of Michigan Health System, Ann Arbor, MI, USA
}

\begin{abstract}
lleal pouch-anal anastomosis is the definitive therapy for ulcerative colitis that is refractory to medical treatment or that has developed neoplasia. Patients with this procedure are routinely followed using directed endoscopic biopsies to monitor for dysplasia in the rectal cuff, residual/recurrent ulcerative colitis, and nonspecific acute inflammation of the ileal pouch (pouchitis), which have different clinical management and outcomes. Thus, accurate localization of mucosal biopsies is crucial to a definitive histological diagnosis, but is complicated by overlapping clinical presentations of pouchitis and ulcerative colitis, post-surgical and inflammatory changes to the mucosa, and altered endoscopic anatomy, resulting in difficulty determining whether a mucosal biopsy is ileal or rectal in origin for both the endoscopist and the pathologist. We explored the utility of CD10 immunohistochemistry to aid diagnosis in this clinical setting by highlighting the enteric mucosa, based on previous studies showing its utility in brush border staining and in the diagnosis of microvillous inclusion disease. We found uniformly positive CD10 immunostaining in normal enteric mucosa, but variable loss of expression in the setting of active enteritis. Specifically, CD10 staining was lost in up to $10 \%$ of the mucosa in $1 / 12$ ileostomies and $4 / 13$ enteric anastomoses, in $10-80 \%$ of the mucosa in $9 / 10$ cases of Crohn's ileitis, in $10-60 \%$ of the mucosa in $7 / 16$ ileal pouches, and in $20-90 \%$ of the mucosa in $6 / 8$ cases of backwash ileitis, usually in the presence of active inflammation. There was no CD10 expression by normal or diseased colonic mucosa. Therefore, while CD10 immunostaining identifies normal enteric mucosa with $100 \%$ specificity, negative staining does not definitively exclude small intestinal mucosa in the setting of active enteritis, a common condition in ileal pouch mucosa.

Modern Pathology (2011) 24, 1627-1632; doi:10.1038/modpathol.2011.122; published online 29 July 2011
\end{abstract}

Keywords: Brush border; CD10; Crohn's disease; lleal pouch; inflammatory bowel disease; pouchitis; ulcerative colitis

Biopsies at or near enterocolic anastomoses are a diagnostic challenge, because post-surgical, inflammatory, and functional changes to the small and large intestinal mucosa can make recognition of the type and location of the sampled mucosa difficult. So-called 'anastomotic site changes' are commonly invoked in such cases, but certain situations require a more definitive diagnosis and discrimination of mucosa type. One such setting is the ileal pouchanal anastomosis, most frequently performed as the

Correspondence: Dr SR Owens, The University of Michigan Health System, Room M5224 Medical Science I, 1301 Catherine Road, Ann Arbor, MI 48109, USA.

E-mail: srowens@umich.edu

Received 17 February 2011; revised 8 June 2011; accepted 8 June 2011; published online 29 July 2011 definitive therapy for ulcerative colitis that is either refractory to medical therapy or which has developed dysplasia or carcinoma. ${ }^{1-3}$

The ileal pouch-anal anastomosis involves an anastomosis of a surgically created ileal reservoiroften termed a 'J-pouch' because of its resemblance to that letter of the alphabet-to what remains of the rectum, with or without a rectal mucosectomy., This results in several potential circumstances of clinical and histological significance. First, the surgically altered anatomy may make endoscopic localization of biopsy site(s) difficult, leading to submission of ileal biopsies labeled as 'rectum' and vice versa. Also, any residual rectal mucosa is subject to persistent inflammation caused by ulcerative colitis (cuffitis) as well as the risk of colitisassociated dysplasia and carcinoma, particularly in 
patients with dysplasia or carcinoma in the resected colon. ${ }^{6-9}$ A rectal mucosectomy may alleviate this somewhat, but in our experience, any colonic/rectal mucosa left behind by such a procedure (or when a mucosectomy is not performed) can interdigitate with the now-adjacent ileal mucosa as the anastomosis matures to create a histological 'hybrid' mucosa that can be difficult to recognize as definitively colonic or enteric under the microscope. Furthermore, the ileal pouch mucosa undergoes a variety of histological and functional changes and is subject to a nonspecific inflammatory reaction known as 'pouchitis', an idiopathic phenomenon that can lead to pain, diarrhea, and bleeding when severe. ${ }^{9-11}$ Finally, the ileal mucosa can potentially be changed by the altered physiology ensuing from its new function as a fecal reservoir. ${ }^{12,13}$

CD10 is a membrane-associated neutral peptidase, also known as neprilysin, enkephalinase, common acute lymphoblastic leukemia antigen, and neutral endopeptidase. ${ }^{14}$ Previous studies have demonstrated its presence on the brush border of small intestinal mucosa and it has been studied and utilized in the diagnosis of microvillous inclusion disease ${ }^{15-18}$ Building on its staining of the enteric brush border, a recent study suggested that CD10 immunohistochemistry can localize biopsies from the ileal pouch and rectal cuff in patients with ileal pouch-anal anastomosis, and utilized it in this way to point out possible errors in endoscopic localization of biopsies from such patients. ${ }^{19}$ Used in this way, the immunostain ostensibly confirms the mucosa as ileal when positive and colonic when negative.

We hypothesized that, while CD10 could potentially be useful as a marker of normal enteric mucosa, alterations in mucosal function and/or ultrastructure may result from surgical anastomosis, the non-physiological function of the ileal pouch as a fecal reservoir, inflammation from pouchitis or other causes, or a combination of these factors, perhaps resulting in a loss or alteration of CD10 expression in the enteric brush border. If CD10 staining was found to be unreliable in inflamed mucosa, its usefulness as a marker of enteric mucosa in the setting of ileal pouch-anal anastomosis, and perhaps more widely in the setting of inflammatory bowel disease and/or other types of enterocolic anastomoses, would be doubtful. We, thus, elected to study the expression of CD10 in normal enteric and colonic mucosa, as well as in a variety of clinical settings that could potentially result in mucosal inflammation, in order to determine the utility of this marker in the identification of mucosal origin on biopsy specimens.

\section{Materials and Methods}

This study was approved by the Institutional Review Board of the University of Pittsburgh
Medical Center. The archives of the Department of Pathology were searched for colonic and enteric specimens sampled in a variety of clinical scenarios, with the goal of identifying normal, surgically altered, and inflamed examples. Both biopsies and resections were included. Histologically normal ileal and colorectal specimens were found by searching for ileocolonic resections removed for colorectal carcinoma. The anatomically altered and inflamed examples of small intestine included resections and biopsies from ileostomies, enteroenteric anastomoses, the enteric side of enterocolic/ ileocolic anastomoses, ileal pouches, cases diagnosed as Crohn's ileitis, and cases diagnosed as 'backwash' ileitis in the setting of ulcerative colitis. Backwash ileitis cases came from patients with active ulcerative colitis that involved the cecum and they invariably had contiguous involvement by active enteritis. However, such cases lacked characteristic findings of Crohn's ileitis such as pyloric gland metaplasia, crypt distortion, and mucosal granulomas. ${ }^{20}$ Inflamed and anatomically altered colonic specimens included ulcerative colitis, Crohn's colitis, the colonic side of enterocolic/ileocolic anastomoses, colostomies, and diversion colitis. Diversion colitis cases came from blind rectal pouches in patients with diverting colostomies and had histological features previously associated with this condition, including prominent lymphoid aggregates and variable amounts of active mucosal inflammation. ${ }^{21} \mathrm{CD} 10$ immunohistochemistry (monoclonal antibody, clone 56C6, Cell Marque, Rocklin, CA) was performed on sections of formalin-fixed, paraffin-embedded tissue from the cases in the departmental immunohistochemistry laboratory, on a Ventana autostainer (Ventana, Tucson, AZ) and according to the manufacturer's instructions.

Because of the potential for histological confusion of mucosa type in the setting of anastomoses as described in the Introduction section, only histologically recognizable mucosa was included in the assessment of CD10 staining. Thus, for anastomotic and ileal pouch-anal anastomosis specimens, only indisputable colonic mucosa, and enteric mucosa with recognizable and irrefutable villous architecture were scored; in the setting of a surgical juxtaposition of colon and small intestine, mucosa that could not be clearly identified as either enteric or colonic was excluded. CD10 staining was scored as positive (complete luminal staining of the brush border on all of the recognizable small intestinal mucosa), negative, or patchy. Histological 'patchiness' could consist of relatively long, contiguous CD10-negative segments or smaller, individually scattered negative areas, but was always recognizable at low- to medium-power microscopic magnification (ie, individually negative epithelial cells or small epithelial cell groups scattered among CD10positive cells were not considered patchy staining). When patchy, the percent of the epithelium with 
loss of staining was independently estimated by both authors at low microscopic power $(\times 4$ magnification) and recorded; areas in which the epithelium was absent (either mechanically stripped or eroded) were not included in this estimation. In addition, the presence or absence of active (neutrophilic) mucosal inflammation was recorded by both authors independently. Finally, after the independent examination by each observer, any discordant results in either stain score or percentage of epithelium with negative staining were resolved by mutual examination and discussion by both observers using a two-headed microscope.

\section{Results}

In total, 103 specimens were retrieved and stained with CD10 (35 colonic and 68 enteric). All 35 colons $(100 \%)$ were negative for CD10, regardless of clinical setting (five normal, six colostomies, five colonic anastomoses, six ulcerative colitis, five diversion colitis, and eight Crohn's colitis), and whether or not they were actively inflamed. All normal small intestines $(6 / 6 ; 100 \%$ ) and normal ileocecal valves $(3 / 3 ; 100 \%)$ had uniformly positive CD10 staining of the enteric mucosa (Figure 1). Surgically altered small intestinal specimens from patients without inflammatory bowel disease had patchy loss of CD10 staining in 5/25 cases $(20 \%)$. Specifically, there were 12 ileostomies, 6 enteroenteric anastomoses, and 7 enterocolic anastomoses, all of which came from patients without inflammatory bowel disease. Of these, one ileostomy (8\%), one enteroenteric anastomosis $(17 \%)$, and three enterocolic anastomoses (43\%) had patchy staining, all with loss of CD10 expression on $\leq 10 \%$ of the epithelium (Figure 2). The ileostomy and the enteroenteric anastomosis with loss of staining both

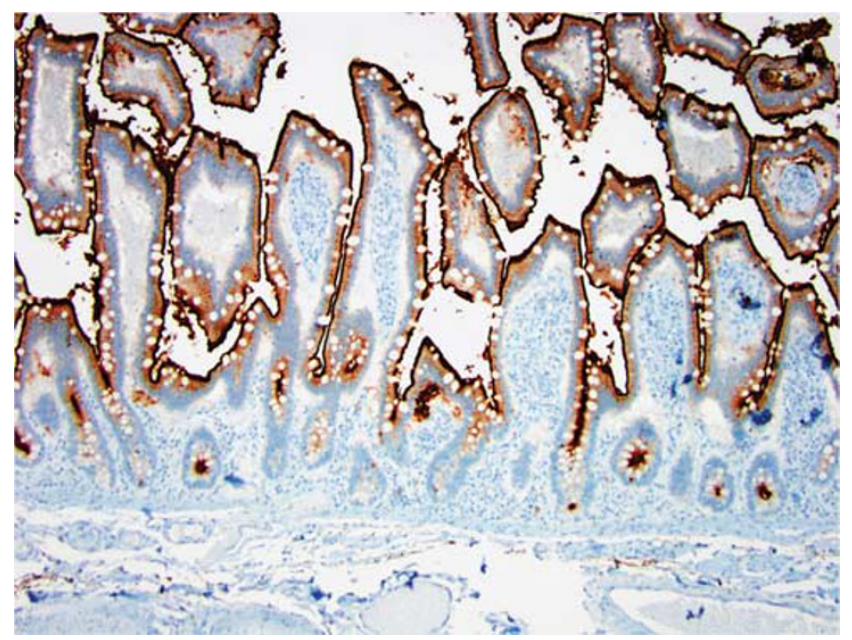

Figure 1 Normal enteric brush border staining with CD10. Note contiguous, strong positivity of the luminal surface of the epithelium. had active inflammation, while one of the three enterocolic anastomoses was actively inflamed.

Specimens from patients with documented inflammatory bowel disease had a higher rate of patchy CD10 staining as well as a larger amount of epithelium that was CD10-negative, with 22/34 $(65 \%)$ losing areas of CD10 positivity. Specifically, 7/16 (44\%) ileal pouches, 6/8 (75\%) backwash ileitis, and 9/10 (90\%) Crohn's ileitis cases had patchy CD10 loss (Figures 3 and 4). All of the cases in which staining was patchy had active mucosal inflammation, and the pattern of loss ranged from

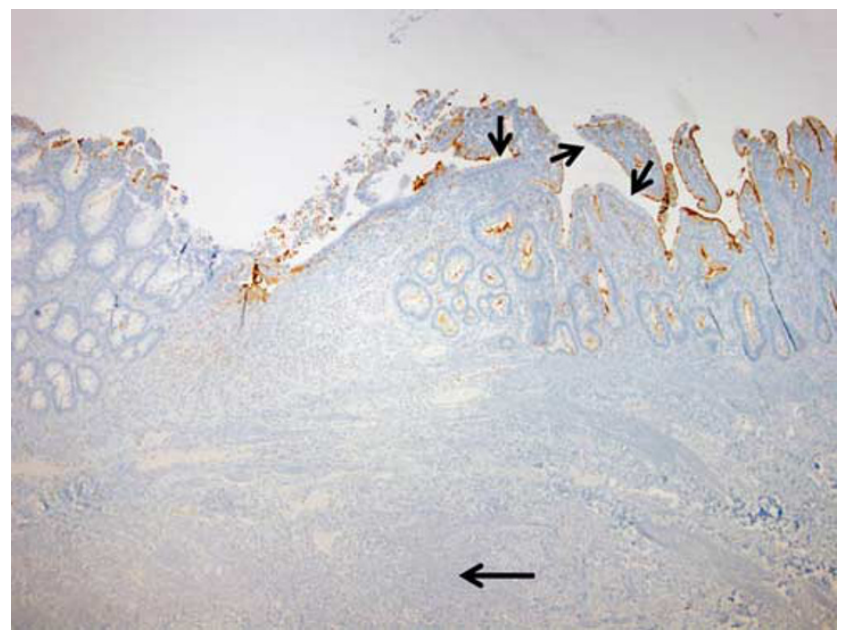

Figure 2 CD10 staining of an enterocolic anastomosis section. The mural scar of the anastomosis can be seen in the bottom portion of the photomicrograph (arrow). The colonic mucosa (left) is uniformly negative for CD10. Most of the enteric mucosa (right) is positive, but there are areas close to the anastomotic junction that are negative (small arrows).

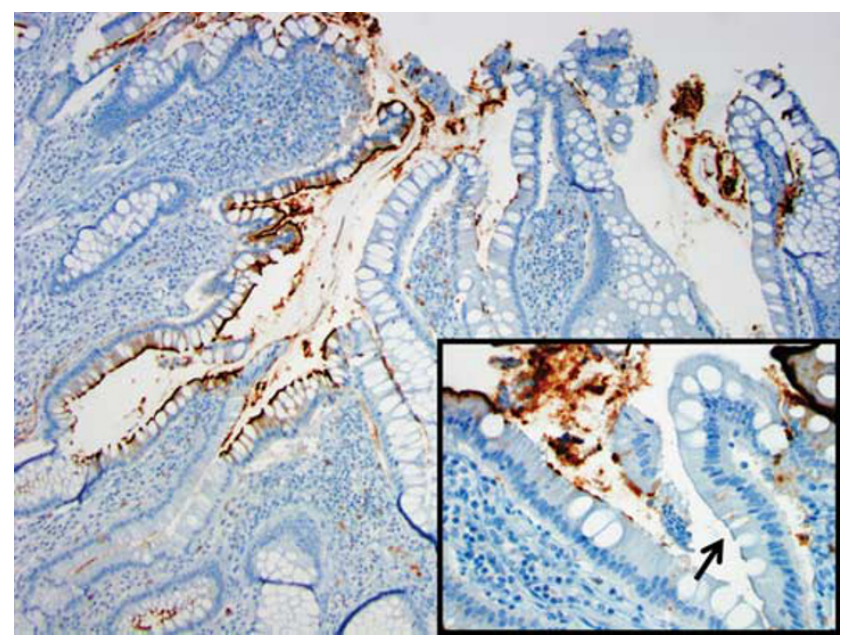

Figure 3 CD10 staining in Crohn's ileitis. This case had an estimated $70 \%$ overall loss of CD10 positivity. Note the patchy nature of the staining, with strong positivity (left) juxtaposed with large negative areas (right). Inset shows high-power view. Note that terminal bar appears to be present even in CD10-negative areas (arrow). 


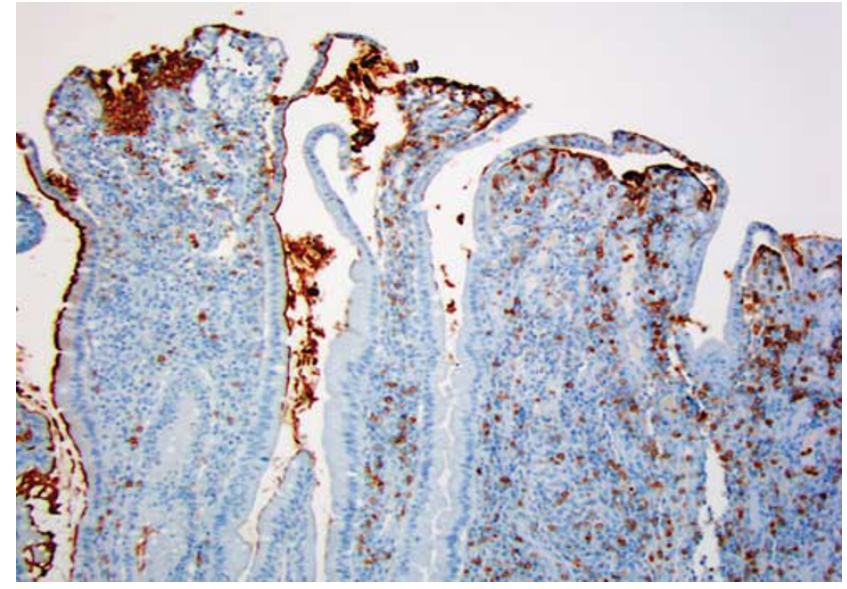

Figure 4 CD10 staining in an ileal pouch that was resected for chronic, treatment-refractory pouchitis. Blunted villi have a mixture of positive staining (left) and almost completely negative epithelium (right).

scattered areas of CD10 negativity to long, contiguous stretches of CD10-negative epithelium, the latter is most common in backwash ileitis cases (Figure 5). The amount of epithelium with loss of staining varied from 20 to $90 \%$ (mean $55 \%$ ) in backwash ileitis, 10 to $60 \%$ (mean $36 \%$ ) in ileal pouches, and 10 to $80 \%$ (mean 32\%) in Crohn's ileitis. In cases with mucosal ulcers, loss of CD10 staining was accentuated in the intact epithelium immediately surrounding the ulcers. Summary results for ileal specimens by diagnostic group are shown in Table 1.

\section{Discussion}

Our findings indicate that CD10 immunohistochemistry is a reliable marker of enteric brush border in normal specimens and is uniformly negative in the colon. Its utility in the setting of active mucosal inflammation and surgical alteration in the small intestine, however, is more limited. Given the variable percentage of lost CD10 expression in actively inflamed enteric mucosa, it is conceivable that entire small biopsies could be CD10-negative and be errantly attributed to having been obtained from the colon, and a negative CD10 staining result in this setting should be interpreted with caution. This is especially true in the setting of inflammatory bowel disease and ileal pouch-anal anastomosisrelated pouchitis, in which the rate and amount of CD10 loss was particularly striking in our series, and is in contrast to the findings reported in what is to our knowledge the only previous study exploring the use of CD10 staining in this setting. ${ }^{19}$

In the clinical setting of ileal pouch-anal anastomosis, directed biopsies of ileal and rectal mucosa are an important part of patient surveillance and management. ${ }^{22}$ Biopsy samples from the ileal pouch

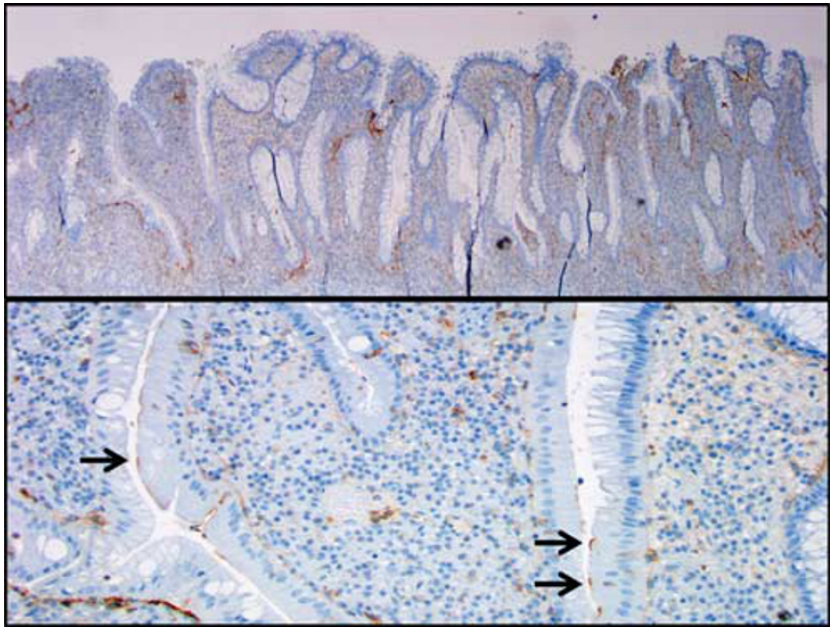

Figure 5 CD10 staining in backwash ileitis. At low power (top), very little if any positivity of the brush border is visible. At high magnification (bottom), tiny patches of positivity (arrows) can be seen.

are important in the diagnosis of active pouchitis, a disease that often responds to conservative therapy with antibiotics and which occurs in a significant number of patients with ileal pouch-anal anastomosis. ${ }^{23,24}$ In contrast, residual or recurrent ulcerative colitis in the rectal cuff may require more aggressive immunosuppression, and rectal mucosa involved by ulcerative colitis remains at risk for the development of colitis-associated dysplasia. Furthermore, the clinical signs and symptoms of active pouchitis have considerable overlap with those of ulcerative colitis, as well as other inflammatory conditions. The accurate assessment of mucosal biopsies from this area is hampered, however, by the alteration of endoscopic anatomy and the histological changes that are potentially induced by changes in ileal function and by inflammation of the pouch and/or rectum. Flattened and inflamed ileal mucosa can strongly resemble biopsy samples of the rectal cuff, and distorted rectal mucosa with a villiform appearance may mimic the ileum. A misclassification of residual ulcerative colitis as 'active pouchitis' could lead to a delay in effective inflammatory bowel disease therapy, while an errant sampling from the ileal pouch, rather than the rectal cuff, diminishes the utility of an endoscopic surveillance examination aimed at detecting rectal dysplasia. In addition, a misclassification of inflamed ileal pouch mucosa as having come from the colon could expose a patient to immunosuppressive therapy when antibiotics for pouchitis may have sufficed. While the known propensity of CD10 to stain the enteric brush border has been suggested to be useful in such situations to determine the origin of difficult-to-interpret biopsies, our data indicate that negative CD10 staining in small biopsy samples may not always equate to an origin in the colon. Thus, though any positive CD10 staining is indicative of the presence of enteric 
Table 1 Results of CD10 staining on ileal specimens, including percentage of epithelium with loss of CD10 expression and presence or absence of active inflammation, arranged by specimen type

\begin{tabular}{|c|c|c|c|}
\hline Specimen & Result & $\%$ Epithelium negative & $\begin{array}{c}\text { Active } \\
\text { inflammation }\end{array}$ \\
\hline \multicolumn{4}{|l|}{ Specimens from patients without IBD } \\
\hline Normal ileum & 6/6 (100\%) Positive & 0 & No \\
\hline Ileocecal valve (ileal mucosa only) & 3/3 (100\%) Positive & 0 & No \\
\hline \multirow[t]{2}{*}{ Ileostomy } & 11/12 (92\%) Positive & 0 & $4 / 11(35 \%)$ \\
\hline & 1/12 (8\%) Patchy & $<10 \%$ & $1 / 1(100 \%)$ \\
\hline \multirow[t]{2}{*}{ Enteroenteric anastomosis } & 5/6 (83\%) Positive & 0 & $2 / 5(40 \%)$ \\
\hline & 1/6 (17\%) Patchy & 10 & $1 / 1(100 \%)$ \\
\hline \multirow{2}{*}{ Enterocolic anastomosis } & 4/7 (57\%) Positive & 0 & No \\
\hline & 3/7 (43\%) Patchy & $<10 \%$ & $1 / 3(33 \%)$ \\
\hline \multicolumn{4}{|l|}{ Specimens from patients with IBD } \\
\hline \multirow[t]{2}{*}{ Crohn's ileitis } & 1/10 (10\%) Positive & 0 & No \\
\hline & 9/10 (90\%) Patchy & $10-80 \%($ mean $=32 \%)$ & $9 / 9(100 \%)$ \\
\hline \multirow{2}{*}{ Ileal pouch } & 9/16 (56\%) Positive & 0 & $8 / 9(89 \%)$ \\
\hline & 7/16 (44\%) Patchy & $10-60 \%($ mean $=36 \%)$ & $7 / 7(100 \%)$ \\
\hline \multirow[t]{2}{*}{ Backwash ileitis } & 2/8 (25\%) Positive & 0 & $2 / 2(100 \%)$ \\
\hline & 6/8 (75\%) Patchy & $20-90 \%($ mean $=55 \%)$ & $6 / 6(100 \%)$ \\
\hline
\end{tabular}

All colonic mucosa was negative for CD10 (data not shown).

mucosa in a biopsy, a negative result cannot be used as confirmation of its absence.

The cases of backwash ileitis in our study merit particular attention. Of the six cases with patchy CD10 staining in this clinical setting, four lost between 20 and $50 \%$ positivity and two lost at least $90 \%$ positivity of the enteric epithelium on the slide. The explanation for this is not clear, but it may reflect a phenotypic alteration of the ileal mucosa, which could be either a result of or a predisposition to, the clinicopathological phenomenon of backwash ileitis. Some observers have suggested that backwash ileitis is a primary ileal disease that occurs in patients with ulcerative colitis, and our observation of markedly decreased CD10 staining in this setting, seemingly out of proportion to that found even in cases of Crohn's disease, may lend weight to this argument. ${ }^{25,26}$ Furthermore, previous studies have suggested that ileal mucosa is altered in other phenotypic ways, such as mucosal permeability and mucin expression, in the setting of backwash ileitis and/or pouchitis, in which a more 'colon-like' mucin pattern has been noted. ${ }^{27,28}$

We have demonstrated a variable loss of brush border CD10 immunostaining in the setting of active enteritis. This finding indicates that, while CD10 is a good marker of enteric mucosa, its use for identification of mucosal origin in the setting of inflammation, such as in ileal pouch-anal anastomosis, is not absolutely reliable, as even a patchy loss of mucosal staining could result in entire fragments of mucosa being falsely interpreted as coming from the colon. Therefore, while any CD10 positivity in a tissue specimen can be used to confirm the presence of enteric mucosa, a negative result does not necessarily indicate that a specimen is colonic. In addition, we found an interesting and relatively extensive loss of CD10 staining in the setting of backwash ileitis, which could be a reflection of phenotypic alteration of the mucosa in this venue as suggested by other observers.

\section{Disclosure/conflict of interest}

The authors declare no conflict of interest.

\section{References}

1 Andersson P, Soderholm JD. Surgery in ulcerative colitis: indication and timing. Dig Dis 2009;27: 335-340.

2 Parks AG, Nicholls RJ. Proctocolectomy without ileostomy for ulcerative colitis. Br Med J 1978;2:85-88.

3 Royston DJ, Warren BF. Are we reporting ileal pouch biopsies correctly? Colorect Dis; published online 19 October 2010; doi: 10.1111/j.1463-1318.2010.02452.x [Epub ahead of print].

4 Chambers WM, Mortensen NJ McC. Should ileal pouch-anal anastomosis include mucosectomy? Colorect Dis 2007;9:384-392.

5 Cohen JL, Strong SA, Hyman NH, et al. Practice parameters for the surgical treatment of ulcerative colitis. Dis Colon Rectum 2005;48:1997-2009.

6 Coull DB, Lee FD, Henderson AP, et al. Risk of dysplasia in the columnar cuff after stapled restorative proctocolectomy. Br J Surg 2003;90:72-75.

7 Das P, Johnson MW, Tekkis PP, et al. Risk of dysplasia and adenocarcinoma following restorative proctocolectomy for ulcerative colitis. Colorect Dis 2007;9: $15-27$.

8 Nilubol N, Scherl E, Bub DS, et al. Mucosal dysplasia in ileal pelvic pouches after restorative proctocolectomy. Dis Colon Rectum 2007;50:825-831.

9 Shen B, Fazio VW, Remzi FH, et al. Clinical approach to diseases of ileal pouch-anal anastomosis. Am J Gastroenterol 2005;100:2796-2807. 
10 de Silva HJ, Millard PR, Kettlewell M, et al. Mucosal characteristics of pelvic ileal pouches. Gut 1991;32:6165.

11 Pardi DS, Shen B. Endoscopy in the management of patients after ileal pouch surgery for ulcerative colitis. Endoscopy 2008;40:529-533.

12 Almeida MG, Kiss DR, Zilberstein B, et al. Intestinal mucosa-associated microflora in ulcerative colitis patients before and after restorative proctocolectomy with an ileoanal pouch. Dis Colon Rectum 2008;51: 1113-1119.

13 Marmorale C, Guercioni G, Siquini W, et al. Evolution of the changes of the ileal pouch mucosa over a long follow-up period. Hepatogastroenterology 2003;50: 1370-1375.

14 Arber DA, Weiss LM. CD10: a review. Appl Immunohistochem 1997;5:125-140.

15 Danielsen EM, Vyas JP, Kenny AJ. A neutral endopeptidase in the microvillar membrane of pig intestine: partial purification and properties. Biochem J 1980; 191:645-648.

16 Groisman GM, Amar M, Livne E. CD10: a valuable tool for the light microscopic diagnosis of microvillous inclusion disease (familial microvillous atrophy). Am J Surg Pathol 2002;26:902-907.

17 Koepsell SA, Talmon G. Light microscopic diagnosis of microvillous inclusion disease on colorectal specimens using CD10. Am J Surg Pathol 2010;34: 970-972.

18 Trejdosiewicz LK, Malizia G, Oakes J, et al. Expression of the common acute lymphoblastic leukaemia antigen (CALLA gp100) in the brush border of normal jejunum and jejunum of patients with coeliac disease. J Clin Pathol 1985;38:1002-1006.
19 Tozbikian GH, Furth EE. Pitfalls in ileal pouch and rectal cuff biopsy analysis: utility of CD10 expression. Mod Pathol 2010;23:170A.

20 Goldstein N, Dulai M. Contemporary morphological definition of backwash ileitis in ulcerative colitis and features that distinguish it from Crohn disease. Am J Clin Pathol 2006;126:365-376.

21 Yeong ML, Bethwaite PB, Prasad J, et al. Lymphoid follicular hyperplasia-a distinctive feature of diversion colitis. Histopathology 1991;19:55-61.

22 Shen B, Remzi FH, Lavery IC, et al. A proposed classification of ileal pouch disorders and associated complication after restorative proctocolectomy. Clin Gastroenterol Hepatol 2008;6:145-158.

23 Cheifetz A, Itzkowitz S. The diagnosis and treatment of pouchitis in inflammatory bowel disease. J Clin Gastroenterol 2004;38(5 Suppl 1):S44-S50.

24 Stocchi L, Pemberton JH. Pouch and pouchitis. Gastroenterol Clin North Am 2001;31:223-241.

25 Abdelrazeq AS, Wilson TR, Leitch DL, et al. Ileitis in ulcerative colitis: is it a backwash? Dis Colon Rectum 2005;48:2038-2046.

26 Haskell H, Andrews CW, Reddy SI, et al. Pathologic features and clinical significance of 'backwash' ileitis in ulcerative colitis. Am J Surg Pathol 2005;29: 1472-1481.

27 Kroesen AJ, Dullat S, Schulzke JD, et al. Permanently increased mucosal permeability in patients with backwash ileitis after ileoanal pouch for ulcerative colitis. Scand J Gastroenterol 2008;43:704-711.

28 Shepherd NA, Jass JR, Duval I, et al. Restorative proctocolectomy with ileal reservoir: pathological and histochemical study of mucosal biopsy specimens. J Clin Pathol 1987;40:601-607. 